\title{
MEASURABLE TRANSFORMATIONS
}

\author{
PAUL R. HALMOS
}

1. Introduction. The purpose of this paper is to review the progress made in the study of measurable and measure preserving transformations during the last 17 years. The interest of mathematicians in this subject was aroused at the end of 1931 by von Neumann's and Birkhoff's proofs of their respective versions of the ergodic theorem $[8,9,101] .{ }^{1}$ It was very quickly recognized that the proper general framework for von Neumann's mean ergodic theorem lay in the direction of Hilbert spaces and Banach spaces, whereas the extent of generality suitable to Birkhoff's theorem was to be found in the concept of a measure space. A measure space is a set possessing no intrinsic algebraic, analytic, or topological structure - all that is necessary is that a concept of measurability and a numerical measure be defined in it. Perhaps the best known nontrivial example of a measure space is one which, to be sure, has many essential non measure theoretic properties, but which may, nevertheless, be considered typical of measure spaces in general-namely the closed unit interval $X=[0,1]$. For the sake of definiteness I shall begin the discussion by considering a one-to-one transformation $T$ of this space $X$ onto itself, such that, for every measurable subset $E$ of $X$, both $T E$ and $T^{-1} E$ are measurable and $\mu(E)=\mu(T E)=\mu\left(T^{-1} E\right.$ ) (where $\mu$ denotes Lebesgue measure in $X$ ). In much of what follows the space $X$ and the transformation $T$ can be replaced by more general spaces and transformations respectively. I shall indicate some of these generalizations in what might be called the geometric direction (that is, generalizations that retain something like an underlying measure space and a transformation acting on it), but I shall not enter at all into the analytic generalizations which constitute the current theory of the mean ergodic theorem.

2. Asymptotic properties. The problems that were first treated, and that are still of interest and importance, are connected with the behavior of the sequence $\left\{T^{n}\right\}$ of powers of $T$. One of the first results in this direction is the Poincaré recurrence theorem, which asserts that, for every measurable set $E$ and for almost every point $x$ in $E$,

An address delivered before the Chicago meeting of the Society on November 26, 1948, by invitation of the Committee to Select Hour Speakers for Western Sectional Meetings; received by the editors November 26, 1948.

${ }^{1}$ Bold face numerals refer to the bibliography at the end. 
there are an infinite number of positive values of $n$ such that $T^{n} x \in E$ $[18,79]$. An equivalent way of phrasing this assertion, in terms of the characteristic function $\chi_{E}$ of $E$, is to say that for every measurable set $E$ the series $\sum_{n=0}^{\infty} \chi_{E}\left(T^{n} x\right)$ diverges for almost every point $x$ in $E$. A related result, proved by Hopf [67], is that if $f$ is any positive measurable function, then the series $\sum_{n=0}^{\infty} f\left(T^{n} x\right)$ diverges for almost every point $x$ in $X$. From the Poincaré recurrence theorem it follows easily that if $E$ is any measurable set of positive measure, then there are an infinite number of positive values of $n$ such that $\mu\left(E \cap T^{n} E\right)>0$, and, conversely, this formulation of Poincarés theorem implies the original one. In this direction the result has been strengthened by Khintchine who showed that if $E$ is any measurable set of positive measure and if $\epsilon$ is any positive number, then the set of values of $n$ for which

$$
\mu\left(E \cap T^{n} E\right)>(\mu(E))^{2}-\epsilon
$$

is relatively dense in the sense of Bohr $[90,126]$.

Birkhoff's ergodic theorem may also be viewed as a statement concerning the recurrence properties of $\left\{T^{n}\right\}$. In its most primitive form the ergodic theorem says that if $E$ is any measurable set and if, for each positive integer $n$ and for every point $x$ in $X, s_{n}(x)$ is the number of values of $i$ for which $T^{i} x \in E, 0 \leqq i \leqq n-1$, then $\lim (1 / n) s_{n}(x)$ $=s^{*}(x)$ exists almost everywhere and, moreover, $\int s^{*}(x) d \mu(x)=\mu(E)$. If the discrete parameter $n$ is interpreted as time, then $s^{*}(x)$ is the relative amount of time (time of sojourn) that $x$ spends in $E$, and the assertion is that the mean sojourn time is almost everywhere defined and that its value depends in an obvious way on the size of $E$. In terms of characteristic functions the statement of the ergodic theorem concerns the existence almost everywhere of $\lim (1 / n) \sum_{i=0}^{n-1} \chi_{E}\left(T^{i} x\right)$ and the value of the integral of the limit function. A more general statement asserts that, for any integrable function $f, \lim (1 / n) \sum_{i=0}^{n-1} f\left(T^{i} x\right)$ $=f^{*}(x)$ exists almost everywhere and that, moreover, $\int f^{*}(x) d \mu(x)$ $=\int f(x) d \mu(x)$.

3. Generalizations. The first attempts to generalize the ergodic theorem consisted of replacing the underlying space $X$ by abstract measure spaces [88] and dropping the hypothesis $\mu(X)<\infty[57,122]$. A slightly more interesting generalization was given by Khintchine [89] who proved that if $f$ is any integrable function on $X$ and $g$ is any periodic function of $n$, then $\lim (1 / n) \sum_{i=0}^{n-1} f\left(T^{i} x\right) g(i)$ exists almost everywhere. The most powerful result in this direction is due to Wiener and Wintner [129] who proved that, if $f$ is, as before, an integrable function, then not only does $\lim (1 / n) \sum_{j=0}^{n-1} e^{2 \pi i \lambda} f\left(T^{j} x\right)$ 
exist almost everywhere for each real number $\lambda$ (here $i=(-1)^{1 / 2}$, but in fact the exceptional set of measure zero can be chosen to be independent of $\lambda$.

The ergodic theorem is a statement about a space, a function, and a transformation. In the preceding paragraph I mentioned the possibility of generalizing the space and the function; there remains the possibility of generalizing the transformation. What, for instance, can be said if the transformation is not necessarily one-to-one, but is still, in one sense or another, measure preserving? Suppose, for the sake of definiteness, that $T$ is a transformation of $X$ onto itself such that the inverse image of every measurable set is a measurable set of the same measure. (A nontrivial example of such a transformation $T$ is defined in the unit interval by $T x=2 x[\bmod 1]$.) If $T$ is such a transformation, if $f$ is any measurable function, and if $g_{n}(x)=f\left(T^{n} x\right)$, $n=0,1,2, \cdots$, then the sequence $\left\{g_{n}\right\}$ has the homogeneity property that the joint $k$-dimensional distribution of any $k$ of its terms depends only on the relative distances between the indices and not on their values. More precisely, it is true that if $A$ is any Borel set in $k$-dimensional space, and if $\left(n_{1}, \cdots, n_{k}\right)$ is any $k$-tuple of distinct non-negative integers, then

$$
\mu\left(\left\{x:\left(g_{n_{1}+m}(x), \cdots, g_{n_{k}+m}(x)\right) \in A\right\}\right)
$$

is independent of $m$. It is a nontrivial extension of the ergodic theorem to say that for any sequence $\left\{g_{n}\right\}$ of integrable functions with this homogeneity property $\lim (1 / n) \sum_{i=0}^{n-1} g_{i}(x)$ exists almost everywhere-this extension is due to Doob [24].

The final generalization that will be mentioned here is one in which the transformation is not required to be measure preserving. The first significant result here was obtained by Hurewicz [73]. I shall state a special case of Hurewicz's theorem-a special case which, however, is typical of the general case and from which, in fact, the general case can easily be derived. Suppose that the transformation $T$ is a one-to-one transformation of a measure space $X$ onto itself such that, for every measurable subset $E$ of $X$, both $T E$ and $T^{-1} E$ are measurable and such that if $\mu(E)=0$, then $\mu(T E)$ $=\mu\left(T^{-1} E\right)=0$. Suppose moreover that $T$ is incompressible in the sense that, for every measurable set $E, E \subset T E$ implies $\mu(E-T E)=0$. It follows from the Radon-Nikodym theorem that, for each integer $n$, there exists a positive measurable function $\omega_{n}$ such that $\mu\left(T^{n} E\right)$ $=\int_{E} \omega_{n} d \mu$ for every measurable set $E$. In the notation so established Hurewicz's ergodic theorem says that, for any integrable function $f$, the weighted averages 


$$
\left(\sum_{i=1}^{n-1} f\left(T^{i} x\right) \omega_{i}(x)\right) /\left(\sum_{i=0}^{n-1} \omega_{i}(x)\right)
$$

converge to a finite limit almost everywhere [42].

4. Indecomposability. If the interval $X$ is the union of two disjoint measurable sets $E$ and $F$ of positive measure, each of which is invariant under the measure preserving transformation $T$, then the study of any property of $T$ on $X$ reduces to the separate studies of the corresponding property of $T$ on $E$ and $T$ on $F$. In such a situation the transformation $T$ may be called decomposable. The most significant transformations are the indecomposable ones-they are usually called metrically transitive [7] or ergodic. In the early days of the theory many special examples of indecomposable transformations were presented in the literature - they occur in fields as apparently diverse as geometry $[51,52,53,54,55,56,66,69,70,71,100,120,121,123,124$, 125], probability [22, 74], and topological groups [39].

For indecomposable transformations the statement of the ergodic theorem can be strengthened by adjoining to it a description of the limit function. Precisely speaking, if $f$ is an integrable function, if $f^{*}(x)=\lim (1 / n) \sum_{i=0}^{n-1} f\left(T^{i} x\right)$, and if the measure preserving transformation $T$ is indecomposable, then $f^{*}(x)$ is equal almost everywhere to a constant, and the value of that constant is $\int f d \mu$. This assertion is at the basis of the celebrated and sometimes misunderstood interchangeability of time means and phase means. It is of interest to observe that the principle of interchangeability is in fact equivalent to indecomposability for measure-preserving transformations on a finite measure space; if, in other words, for each integrable function $f, f^{*}$ is equal almost everywhere to a finite constant, then $T$ is indecomposable $[103]$.

A question might be raised as to the extent to which Birkhoff's theorem could be extended to functions which are not necessarily integrable. It is clear that if a transformation is sufficiently decomposable (meaning that there exists a disjoint infinite sequence of measurable invariant sets of positive measure), then the conclusion of Birkhoff's theorem is true for many non integrable functions. The identity transformation is from this point of view the extreme caseif $T$ is the identity, then the conclusion of Birkhoff's theorem is true for every function. For indecomposable transformations $T$, on the other hand, it can be proved that if $f$ is a non-negative measurable function, or, more generally, a measurable function with the property that either its positive part or its negative part is integrable, and if $\lim (1 / n) \sum_{i=0}^{n-1} f\left(T^{i} x\right)$ exists and is finite almost everywhere, then $f$ 
must be integrable. M. Gerstenhaber has recently shown me an example which proves that for arbitrary measurable functions this result is not necessarily true.

5. Decompositions. A natural problem in connection with the concept of indecomposability is whether or not every measure preserving transformation may be decomposed into indecomposable components. In order to clarify the question and motivate the answer it is helpful to consider an example. Suppose that the measure space $X$ is the unit square in the Cartesian plane, and that the measure preserving transformation $T$ on $X$ has the property that it leaves unaltered the first coordinate of each point. This implies that every subset $E$ of $X$ which depends on the first coordinate alone, that is, every set $E$ which is the union of a class of vertical segments, is invariant under $T$. Suppose moreover that the invariant sets so obtained exhaust essentially all possibilities - that is, that every measurable invariant set is, modulo sets of measure zero, a union of vertical segments. Since each vertical segment may be considered as a measure space on its own right (with linear Lebesgue measure) and since, in the presence of the conditions described above, the transformation $T$ is indecomposable on each such segment, this situation is an example of a decomposable transformation which is in a certain intuitively obvious sense made up of many little indecomposable components.

The general situation exemplified by the preceding paragraph may be described as follows. Suppose that to each point $x$ of a measure space $X$ (with measure $\mu$ ) there corresponds a measure space $Y_{x}$ (with measure $\mu_{x}$ ) so that the spaces corresponding to distinct points are disjoint. Suppose that a concept of measurability and a numerical measure $\lambda$ are introduced into the set $Z$ of all those pairs $(x, y)$ for which $x \in X$ and $y \in Y_{x}$, in such a way that whenever $A$ is a measurable subset of $X$, then the set $A^{*}=\{(x, y): x \in A\}$ is a measurable subset of $Z$. If, for every measurable subset $E$ of $Z$,

$$
\lambda(E)=\int_{X} \mu_{x}\left(E \cap Y_{x}\right) d \mu(x),
$$

then the measure space $Z$ (with measure $\lambda$ ) is called a direct sum of the measure spaces $Y_{x}$ with respect to the measure space $X$. The best possible theorem on the decomposability of a measure preserving transformation $T$ on a measure space $Z$ would presumably assert that $Z$ may be represented as a direct sum in such a way that the class of measurable invariant sets coincides, except possibly for sets of 
measure zero, with the class of all sets of the form $A^{*}$; this would imply that the transformation $T$ on $Y_{x}$ is indecomposable for almost every $x$ in $X$. The first theorem of this type (in case $Z$ is a complete metric space) was proved by von Neumann [103]; extensions to more general spaces were later given by Dieudonné [21] and myself [36, 44].

6. Density and category theorems. How likely is a measure preserving transformation to be indecomposable? Birkhoff conjectured that in some sense the indecomposable case is the general case. One possible way of establishing this conjecture was to introduce a suitable metric or topology into the set $\boldsymbol{T}$ of all measure preserving transformations (after identifying two transformations which differ only on a set of measure zero) and then to show that the subset $D$ of decomposable transformations is of the first category. This was first done by Oxtoby and Ulam for measure preserving homeomorphisms of certain subsets of Euclidean spaces and later by me for arbitrary measure preserving transformations $[40,107]$. These topological investigations had some interesting byproducts of which at least one is worth mentioning. A class of particularly simple measure preserving transformations of the interval is obtained by dividing the interval into a finite number of subintervals of equal length and sending each such interval into another such interval by translation. The assertion concerning the class $\boldsymbol{P}$ of transformations so obtained is that it is everywhere dense in $T$-in other words every measure preserving transformation is the limit of a sequence of permutations of intervals.

7. Strong mixing. It is possible to define the concept of indecomposability of a transformation $T$ in terms of the asymptotic behavior of the sequence of powers of $T$. It is in fact an easy consequence of the ergodic theorem that if $T$ is indecomposable, then

$$
\lim \frac{1}{n} \sum_{i=0}^{n-1} \mu\left(E \cap T^{i} F\right)=\mu(E) \mu(F)
$$

for every pair of measurable sets $E$ and $F$ and that, conversely, the validity of this relation for every pair of measurable sets implies that $T$ is indecomposable. Since the condition $\mu(E \cap F)=\mu(E) \mu(F)$ is the usual requirement in the definition of independence in the sense of the theory of probability, the equation $\left(^{*}\right)$ may be viewed as asserting that asymptotically, in the sense of Cesaro convergence, any two measurable sets, of which one is held fixed and the other is allowed to move under the influence of the transformation $T$, tend to become independent of each other. 
The equation $\left(^{*}\right)$ has another natural physical interpretation. Suppose that the transformation $T$ is visualized as a particular way of stirring a container (of total volume 1) full of an incompressible fluid which may be thought of as 99.44 per cent water and .56 per cent red ink. If $F$ is the region occupied by the red ink, then, for any part $E$ of the container, the relative amount of red ink in $E$, after $n$ repetitions of the act of stirring, is given by $\mu\left(E \cap T^{n} F\right) / \mu(E)$. The indecomposability of the transformation $T$ implies therefore that on the average this relative amount of redness is exactly equal to .56. In general, in physical situations like this one, one expects to be justified in making a much stronger statement, namely that, after the liquid has been stirred sufficiently often, every part $E$ of the container will contain approximately .56 per cent red ink. In mathematical language this pious hope amounts to replacing Cesaro convergence by ordinary convergence, that is, replacing $\left(^{*}\right)$ by

$$
\lim \mu\left(E \cap T^{n} F\right)=\mu(E) \mu(F) .
$$

Transformations $T$ satisfying (**) for every pair $E$ and $F$ of measurable sets are called strongly mixing.

It may be worth while to give an example of a strongly mixing transformation on the interval $X$. The definition of such a transformation is surprisingly simple. For each $x$ in $X$, let $x=\sum_{i=1}^{\infty} a_{i} / 2^{i}$ be the binary expansion of $x$, and let $p$ be a cyclic permutation of the set of all positive integers; for instance $p$ may be defined by

$$
\begin{aligned}
p(1)=2, p(2 n)=2 n+2, \text { and } p(2 n+1)=2 n & -1, \\
n & =1,2, \cdots .
\end{aligned}
$$

If $S$ is defined by $S x=\sum_{i=1}^{\infty} a_{p(i)} / 2^{i}$, then, except for some easily rectifiable trouble caused by the non uniqueness of the binary expansion, it follows that $S$ is one-to-one from $X$ onto $X$ and measure preserving. Strongly mixing transformations similar to this one occur frequently in probability theory.

8. Weak mixing. Between indecomposable transformations and strongly mixing transformations there is room for another conceptthe concept of a weakly mixing transformation. This apparently artificial concept is of great technical significance. A measure preserving transformation $T$ is, by definition, weakly mixing if

$$
\lim \frac{1}{n} \sum_{i=0}^{n-1}\left|\mu\left(E \cap T^{i} F\right)-\mu(E) \mu(F)\right|=0
$$

for every pair $E$ and $F$ of measurable sets. In mathematical language, 
the definition of weak mixing substitutes strong Cesaro convergence for the Cesaro convergence occurring in the definition of indecomposability and for the ordinary convergence occurring in the definition of strong mixing. It is an analytic exercise to show that $\left({ }^{* * *}\right)$ is satisfied if and only if there exists a set $N$ of positive integers such that $N$ has density zero and such that

$$
\lim _{n \in N} \mu\left(E \cap T^{n} F\right)=\mu(E) \mu(F) .
$$

If indecomposability is expressed by saying that on the average $E$ is .56 per cent red, and if strong mixing is expressed by saying that after a while $E$ will be .56 per cent red, then weak mixing can be expressed by saying that after a while $E$ will be .56 per cent red, with the exception of a few rare instants during which it may be either too scarlet or else too pale a pink.

For any two measure preserving transformations $S$ and $T$, the direct product $S \times T$ is defined as that transformation on the Cartesian product of the space $X$ with itself which sends each point $(x, y)$ into $(S x, T y)$. The first indication that weak mixing is more than an analytic artificiality is in the assertion that $T$ is weakly mixing if and only if its direct product with itself is indecomposable $[65,83,84]$.

The physical intuition which motivated the conjecture that in general a measure preserving transformation is indecomposable seems also to indicate that in general a measure preserving transformation is weakly and even strongly mixing. With the usual (category) interpretation of the phrase "in general," I proved that this conjecture is right for weak mixing [41]. In a recent paper Rokhlin [115] showed, by a very simple and elegant argument, that for strong mixing on the other hand the conjecture is just as wrong as it can be, and that, in fact the set $\boldsymbol{S}$ of strongly mixing transformations is a set of the first category in the set $T$ of all measure preserving transformations.

9. Automorphisms and unitary operators. A measure preserving transformation $T$ on a measure space $X$ induces in an obvious way an automorphism of the measure algebra of measurable sets modulo sets of measure zero, or, in other words, a set transformation which assigns to each class of sets, any two members of which differ only on a set of measure zero, another such class. The question of whether or not the converse is true has received some attention [38, 102]. The answer is yes except in pathological measure spaces. Since this pathology is not of very much interest from the point of view of this paper, I shall from now on require that the space $X$ be non patho- 
logical in this sense. It has been proved that all the well known measure spaces satisfy this requirement, that is, that for them every automorphism of the measure algebra of measurable sets modulo sets of measure zero is indeed induced by a one-to-one measure preserving transformation of the space onto itself.

Another, very similar, problem is suggested by the consideration of the complex Hilbert space $L_{2}$. If, for each $f$ in $L_{2}$, an element $U f$ in $L_{2}$ is defined by $(U f)(x)=f(T x)$, then the fact that $T$ is measure preserving implies by an easy and familiar argument (proceeding through finite linear combinations of characteristic functions of measurable sets) that

$$
\|U f\|^{2}=\int|f(T x)|^{2} d \mu(x)=\int|f(x)|^{2} d \mu(x)=\|f\|^{2} .
$$

Since the transformation $U$ is a one-to-one linear transformation of $L_{2}$ onto itself, the last written relation means that $U$ is unitary [93, 133]. In other words, to each measure preserving transformation $T$ on $X$ there corresponds a unitary operator $U$ on $L_{2}$; the first problem that has to be settled is the characterization of the unitary operators that can arise in this way. A more or less satisfactory answer is known: a unitary operator is induced by a measure preserving transformation $T$ in the way just now outlined if and only if it sends each bounded measurable function into a bounded measurable function and is such that, for any two bounded measurable functions $f$ and $g, U(f g)$ $=U f \cdot U g$, where the indicated multiplications denote the pointwise product of the factors [103].

It is natural to hope that at least some of the measure theoretic properties of $T$ can be described in the language of Hilbert space and that, conversely, the ideas suggested by the Hilbert space point of view may have measure theoretic significance. There is a fact quite near the surface which seems at least partially to fulfill this hope: a necessary and sufficient condition that $T$ be indecomposable is that the complex number 1 be a simple proper value of $U$ [103]. (A proper function of proper value 1 is of course simply an invariant function. Each function in the one-dimensional family of constant functions is invariant; the theorem just stated characterizes indecomposability by the absence of any other invariant functions.) A considerably deeper fact, known as the mixing theorem, is that $T$ is weakly mixing if and only if 1 is a simple proper value of $U$ and moreover $U$ has no other proper values $[62,63,65,94]$.

10. Isomorphism and pure point spectrum. A fundamental prob- 
lem of the theory of measurable transformations is the problem of isomorphism. Two measure preserving transformations $T_{1}$ and $T_{2}$ are called isomorphic if there exists a measure preserving transformation $T$ which carries $T_{1}$ into $T_{2}$, that is, for which $T T_{1} T^{-1}=T_{2}$. An obvious solution of the isomorphism problem is suggested by the first result mentioned in the preceding section-two measure preserving transformations are isomorphic if and only if the automorphisms they induce are conjugate elements in the group $\boldsymbol{T}$ of all automorphisms.

One might make the more promising and less trivial conjecture that all the measure theoretic properties of a transformation $T$ are reflected by the operatorial properties of its unitary operator $U$, that is, that $T_{1}$ and $T_{2}$ are isomorphic if and only if the corresponding unitary operators $U_{1}$ and $U_{2}$ are spectrally equivalent, or, in other words, if and only if there exists a unitary operator $U$ for which $U U_{1} U^{*}=U_{2}$.

There is an interesting class of measure preserving transformations for which the conjecture expressed in the preceding paragraph is true. In order to motivate the introduction of this class, let the measure space $X$ be a compact abelian group (with Haar measure) and let $\alpha$ be any fixed element of $X$. If $T$ is defined, for every $x$ in $X$, by $T x=x+\alpha$, then $T$ is a measure preserving transformation of $X$ onto itself. If $\phi$ is any character of the group $X$, then the equation $\phi(x+\alpha)$ $=\phi(\alpha) \phi(x)$ shows that $\phi$ is a proper function of the unitary operator $U$ corresponding to $T$, with proper value $\phi(\alpha)$. Since the set of all characters is a complete orthonormal set in $L_{2}$, the transformation $T$ is said to have pure point spectrum - the general definition of a measure preserving transformation with pure point spectrum is that the set of proper functions of its induced unitary operator is large enough to contain a complete orthonormal set. The conjecture concerning the relation of isomorphism and spectral equivalence is true in this sense: two indecomposable measure preserving transformations with pure point spectrum are isomorphic if and only if their induced unitary operators are spectrally equivalent - this was proved by von Neumann [103]. In this connection it should also be mentioned that every indecomposable measure preserving transformation with pure point spectrum is known to be of the type described in the example above-that is, each such transformation is necessarily a translation by a suitable element in a suitable compact abelian group [38].

11. Isomorphism and mixed spectrum. The result of the preceding section is not true for arbitrary measure preserving transformationsit is possible, in other words, to construct an example of two measure 
preserving transformations $T_{1}$ and $T_{2}$ such that $T_{1}$ and $T_{2}$ are not isomorphic but such that the corresponding unitary operators are spectrally equivalent. This construction has not been published so far-it is the result of joint work by von Neumann and myself. I proceed to sketch the details of the theory behind the construction.

With each measure preserving transformation $T$ it is possible to associate a sequence $\left\{\boldsymbol{G}_{n}\right\}$ of classes of bounded measurable functions as follows. The initial class $G_{0}$ contains only the function which is identically equal to 1 ; for $n \geqq 1$, the class $G_{n}$ consists of those functions $f$ which satisfy almost everywhere an equation of the form $f(T x)=g(x) f(x)$, with $g \in G_{n-1}$. The class $G_{1}$ is then the class of invariant functions; if $T$ is indecomposable, so that $G_{1}$ is the class of constant functions, then $G_{2}$ is the class of proper functions; for positive values of $n$ the functions of $G_{n}$ may be viewed as generalized proper functions belonging to proper values which instead of being necessarily constants are elements of $G_{n-1}$. It may happen that, for a suitable positive integer $n, G_{n}=G_{n+1}$ (and hence $G_{n}=G_{n+p}$ for every positive integer $p$ ); the least positive integer for which this happens is then denoted by $n(T)$. If for instance $T$ is weakly mixing, then (since $T$ is a fortiori indecomposable) $G_{1}$ is the class of constant functions and therefore, by the mixing theorem, $n(T)=1$.

An example which shows that isomorphism is not the same as spectral equivalence is now easy to construct. Let the measure space be the torus represented as all pairs $(x, y)$ of real numbers modulo 1 . If $T_{1}(x, y)=(x+\alpha, y+x) \quad[\bmod 1]$, and if $T_{2}(x, y)=(x+\alpha, S y)$ [mod 1], where $\alpha$ is an irrational number and $S$ is the transformation described in $\$ 8$ as an example of a strongly mixing transformation, then a reasonably straightforward calculation shows that the induced unitary operators are spectrally equivalent. The proof that, nevertheless, $T_{1}$ and $T_{2}$ are not isomorphic leans on the concepts introduced in the preceding paragraph; it can in fact be shown that $n\left(T_{1}\right)=3$ and $n\left(T_{2}\right)=2$.

12. Invariant measures. With the exception of the ergodic theorem, most of the considerations in the preceding sections deal with measure preserving transformations. How restrictive is the requirement that a transformation preserve some measure? One of the best precise and nontrivial formulations of this question is the following one. Suppose that $T$ is a one-to-one transformation of a measure space $X$ onto itself such that for every measurable subset $E$ of $X$ both $T E$ and $T^{-1} E$ are measurable and such that if $\mu(E)=0$, then $\mu(T E)$ $=\mu\left(T^{-1} E\right)=0$; does there then exist a measure $\lambda$ on the class of all measurable subsets of $X$ such that $\lambda(E)$ vanishes if and only if $\mu(E)$ 
vanishes, such that $X$ is the union of countably many measurable sets on each of which $\lambda$ is finite, and such that $\lambda(T E)=\lambda(E)$ for every measurable set $E$ ?

An interesting necessary and sufficient condition for the existence of a finite $\lambda$ was given by Hopf [64]. In order to describe this condition it is necessary to introduce some new concepts. Two measurable sets $E$ and $F$ are primitively equivalent with respect to a transformation $T$ if there exists an integer $n$ such that $T^{n} E=F$. Two measurable sets $E$ and $F$ are equivalent with respect to a transformation $T$ if (after possibly omitting a set of measure zero from both of them) both $E$ and $F$ may be written as unions of disjoint sequences of measurable sets so that each term in the sequence representing $E$ is primitively equivalent to the corresponding term in the sequence representing $F$. In imitation of Dedekind's definition of finiteness, it is customary to say that a measurable set $E$ is bounded (or finite) with respect to $T$ if whenever $E$ is equivalent to a subset $F$ of itself, then $\mu(E-F)=0$. Hopf's condition for the existence of a finite invariant measure is that $X$ be bounded.

The concept of boundedness, while it gives quite a bit of insight into the structure of measurable transformations, is not very easy to apply. Until recently, for example, it was not known whether or not the condition was vacuously satisfied, that is, whether or not there existed any transformations at all which did not satisfy it. In 1947 I succeeded in showing that such a transformation does exist and hence that the search for invariant measures must be conducted among measures which are allowed to take infinite values [43]. The proof is based on the fact that it is sufficient to exhibit an indecomposable measure preserving transformation on a non atomic measure space of infinite measure; the first transformation with these properties was constructed by Oxtoby. The condition of Hopf has a natural extension to the infinite case, but is then even harder to apply [43, 85 ]. While it seems plausible to conjecture that an invariant measure need not always exist, the question is still open. Either an example or, if the conjecture is wrong, a general existence theorem would be of considerable interest. In the meantime the theory of measurable but not necessarily measure preserving transformations deserves a little investigation. The first steps of such an investigation were carried out by Rademacher in 1916 [110] and a few fragmentary results have been obtained since then $[43,46]$.

13. Flows. Virtually all the problems discussed above for a single transformation $T$ and its iterates $T^{n}, n=0, \pm 1, \pm 2, \cdots$, make sense for a one-parameter family of transformation $\left\{T_{t}\right\},-\infty<t$ 
$<+\infty$, which have the group property $T_{t+s}=T_{t} T_{s}$ for all $t$ and $s$. Such a family of measure preserving transformations is called a flow; a flow is called measurable if $T_{t} x$ is a measurable function of $(x, t)$. If sums are replaced by integrals and the problems of measurability receive a modicum of attention, then the ergodic theorem, the mixing theorem, the decomposition into indecomposable parts, and many other essential results can be extended from transformations to flows without any new conceptual difficulties.

There is, however, at least one notion which is suggested by the study of flows and not by the automatic process of generalization. This is the concept of a flow built under a function. Suppose that $f$ is any positive measurable function on a measure space $X$ and that $T$ is any one-to-one measure preserving transformation of $X$ onto itself. Let $\bar{X}$ be the ordinate set of $f$, that is, $\bar{X}$ is a subset of the product space of $X$ with the real line and is defined by

$$
\bar{X}=\{(x, t): 0 \leqq t<f(x)\} .
$$

For convenience of language conceive $X$ as a horizontal interval, and the parameter $t$ as time; it will then make sense to speak of points of $\bar{X}$ going "up" with a certain "velocity." Let each point $(x, t)$ of $\bar{X}$ move up, in this sense, with a uniform unit velocity, until it hits the "ceiling" $f(x)$; let it then be put back at the place $(T x, 0)$ and continue on up from there. This procedure defines a flow in $\bar{X}$; the flow so defined is called the flow built on $T$ under $f$. The study of such a flow may obviously be reduced to the study of the transformation $T$ and the function $f$; it is consequently a very useful thing to know that every measurable indecomposable flow is isomorphic to a flow built under a function. This result was proved by Ambrose [2] and later extended to all non pathological flows by Ambrose and Kakutani [3].

It is interesting to observe that the concept of a flow built under a function has a discrete analogue which promises to be of interest even in the study of a single transformation. Let $f$ be a measurable function from a measure space $X$ to the set of non-negative integers, and write $E_{n}=\{x: f(x) \geqq n\}, n=0,1,2, \cdots$. The set $\bar{X}$ of all pairs $(x, n)$, where $n=0,1,2, \cdots$ and $x \in E_{n}$, is an analogue of what in the continuous case was called the ordinate set of $f$. If $T$ is a transformation on $X$, then a transformation $\bar{T}$ may be defined on $\bar{X}$ by setting $\bar{T}(x, n)=(x, n+1)$ whenever $x \in E_{n+1}$ and $\bar{T}(x, n)=(T x, 0)$ otherwise. The example (mentioned in the preceding section) of a transformation which does not preserve any suitable finite measure was constructed with the aid of such methods; a systematic study of 
the relation between $T$ and $\bar{T}$ was recently begun by Kakutani [82].

14. Unsolved problems. In connection with the discussion above I have already had occasion to mention two or three unsolved problems. The most important one among these is the problem of the existence of invariant measures. In this section I shall mention a few more directions in which further progress would be desirable.

Quite a few of the results discussed above, but by no means all, can be extended to measure spaces of not necessarily finite measure; a systematic investigation of measure preserving transformations on infinite measure spaces is still lacking. Another obvious problem is to extend the ergodic theorem (known for the additive group of the integers and the additive group of the real numbers) to more general groups of transformations; some results in this direction are known for finite dimensional vector groups $[109,127,128]$.

In order to state the next problem, it is convenient to introduce one more concept. If, for each of a countable set of indices $i, X_{i}$ is a measure space with measure $\mu_{i}$ such that $\mu_{i}\left(X_{i}\right)=1$, then it is possible to make the Cartesian product $X$ of all the $X_{i}$ into a measure space in a way which is a natural extension of the concept of Cartesian product for a finite number of spaces. Such product spaces are well known in probability theory. Suppose in particular that the spaces $X_{i}$ are all equal to a fixed space $X_{0}$ and that the domain of the index $i$ is the set of all integers. A point $x$ of $X$ is in this case a sequence, $x=\left(\cdots, x_{-2}, x_{-1}, x_{0}, x_{1}, x_{2}, \cdots\right), x_{i} \in X_{0}$. If a transformation $T_{0}$ is defined on $X$ by $T_{0} x=\left(\cdots, y_{-2}, y_{-1}, y_{0}, y_{1}, y_{2}, \cdots\right)$, $y_{n}=x_{n+1}$, then this coordinate shift $T_{0}$ is a one-to-one measure preserving transformation of $X$ onto itself. If $X_{0}$ is the real line (with a suitable measure $\mu_{0}$ satisfying $\mu_{0}\left(X_{0}\right)=1$ ) and if the function $f_{0}$ on $X$ is defined by $f_{0}(x)=x_{0}$, then results much more precise than the ergodic theorem are known about the asymptotic behavior of the sequence $\left\{f_{0}\left(T_{0}^{n} x\right)\right\}$. It would be of interest to obtain analogues of these results (for example, the law of the iterated logarithm and the central limit theorem) for a wider class of transformations and functions than the ones here described.

An attempt in this direction has been made by Izumi [75]. Motivated by the fact that (under a mild restriction on the measure $\mu_{0}$ )

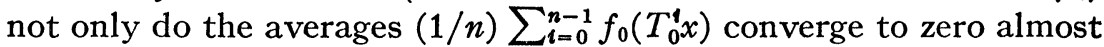
everywhere but even the series $\sum_{n=1}^{\infty}(1 / n) f_{0}\left(T_{0}^{n} x\right)$ is convergent almost everywhere, Izumi formulated a set of conditions on the measure preserving transformation $T$ (and on the function $f$ ) sufficient to ensure the convergence almost everywhere of $\sum_{n=1}^{\infty}(1 / n) f\left(T^{n} x\right)$. Unfortunately Izumi's requirements turned out to be so stringent 
that the only transformation which can satisfy them is the identity transformation on a measure space consisting of exactly one point [45].

In conclusion I emphasize again that the isomorphism problem is almost completely untouched in most nontrivial cases. It is, of course, hard to formulate precisely what is meant by the injunction: "find necessary and sufficient conditions for the isomorphism of two measure preserving transformations." There are, however, many concrete examples of measure preserving transformations concerning which it is not known whether or not any two are isomorphic-the vague task of finding a complete set of invariants can at least at the beginning be replaced by the specific task of finding sufficiently many invariants to sort out into isomorphism classes these known examples [39]. If, for instance, the coordinate shift on the Cartesian product of countably many $k$-point spaces (in which each point has measure $(1 / k))$ is denoted by $T_{k}$, then is $T_{2}$ isomorphic to $T_{3}$ or is it not? Nobody knows.

\section{BIBLIOGRAPHY}

The following is a list, as nearly complete as I could make it, of the papers and books directly relevant to the theory of measurable and measure preserving transformations. It does not contain works concerning applications to physics or stochastic processes, generalizations to Markoff chains or mean ergodic theorems, and analytical or topological results about dynamical systems.

1. W. Ambrose, Change of velocities in a continuous ergodic flow, Duke Math. J. vol. 8 (1941) pp. 425-440. 739.

2. - Representation of ergodic flows, Ann. of Math. vol. 42 (1941) pp. 723-

3. W. Ambrose and S. Kakutani, Structure and continuity of measurable flows, Duke Math. J. vol. 9 (1942) pp. 25-42.

4. W. Ambrose, P. R. Halmos, and S. Kakutani, The decomposition of measures, II, Duke Math. J. vol. 9 (1942) pp. 43-47.

5. M. Bebutoff and W. Stepanoff, Sur le changement du temps dans les systèmes dynamiques possédant une mesure invariante, C. R. (Doklady) Acad. Sci. URSS vol. 24 (1939) pp. 217-219.

6. - Sur la mesure invariante dans les systèmes dynamiques qui ne diffèrent que par le temps, Rec. Math. (Mat. Sbornik) N.S. vol. 7 (1940) pp. 143-166.

7. G. D. Birkhoff and P. A. Smith, Structure analysis of surface transformations, J. Math. Pures Appl. vol. 7 (1928) pp. 345-379.

8. G. D. Birkhoff, Proof of a recurrence theorem for strongly transitive systems, Proc. Nat. Acad. Sci. U.S.A. vol. 17 (1931) pp. 650-655.

9. - Proof of the ergodic theorem, Proc. Nat. Acad. Sci. U.S.A. vol. 17 (1931) pp. 656-660.

10. G. D. Birkhoff and B. O. Koopman, Recent contributions to ergodic theory, Proc. Nat. Acad. Sci. U.S.A. vol. 18 (1932) pp. 279-282. 
11. G. D. Birkhoff, Probability and physical systems, Bull. Amer. Math. Soc. vol. 38 (1932) pp. 361-379.

12. - Some unsolved problems of theoretical dynamics, Science vol. 94 (1941) pp. $598-600$.

13. - What is the ergodic theorem?, Amer. Math. Monthly vol. 49 (1942) pp. $222-226$.

14. - The ergodic theorems and their importance in statistical mechanics, Revista de Ciencias vol. 44 (1942) p. 251.

15. N. Bogoliouboff and N. Kryloff, Les mesures invariantes et la transitivité, C. R. Acad. Sci. Paris vol. 201 (1935) pp. 1454-1456.

16. - Les mesures invariantes et transitives dans la mécanique non linéaire, Rec. Math. (Mat. Sbornik) N.S. vol. 1 (1936) pp. 707-711.

' 17. - La théorie générale de la mesure dans son application à l'étude des systèmes dynamiques de la mécanique non linéaire, Ann. of Math. vol. 38 (1937) pp. 65-113.

18. C. Carathéodory, Über den Wiederkehrsatz von Poincarê, Sitzungsberichte der Preussischen Akademie der Wissenschaften vol. 32 (1919) pp. 580-584.

19. - Bemerkungen zum Riesz-Fischerschen Satz und zur Ergodentheorie, Abh. Math. Sem. Hansischen Univ. vol. 14 (1941) pp. 351-389.

20. —- Bemerkungen zum Ergodensatz von G. Birkhoff, Sitzungsberichte der Mathematisch-Naturwissenschaftlichen Klasse der Bayerischen Akademie der Wissenschaften zu München (1944) pp. 189-208.

21. J. Dieudonné, Sur le théorème de Lebesgue-Nikodym (III), Annales de l'Université de Grenoble vol. 23 (1948) pp. 25-53.

22. J. L. Doob, Probability and statistics, Trans. Amer. Math. Soc. vol. 36 (1934) pp. 759-775.

23. - One-parameter families of transformations, Duke Math. J. vol. 4 (1938) pp. 752-774.

24. - The law of large numbers for continuous stochastic processes, Duke Math. J. vol. 6 (1940) pp. 290-306.

25. J. L. Doob and R. A. Leibler, On the spectral analysis of a certain transformation, Amer. J. Math. vol. 65 (1943) pp. 263-272.

26. Y. N. Dowker, Invariant measure and the ergodic theorems, Duke Math. J. vol. 14 (1947) pp. 1051-1061.

27. N. Dunford, Spectral theory. I. Convergence to projections, Trans. Amer. Math. Soc. vol. 54 (1943) pp. 185-217.

28. - Spectral theory, Bull. Amer. Math. Soc. vol. 49 (1943) pp. 637-651.

29. N. Dunford and D. S. Miller, On the ergodic theorem, Trans. Amer. Math. Soc. vol. 60 (1946) pp. 538-549.

30. K. Fan, Les fonctions asymptotiquement presque-périodiques d'une variable entière et leur application à l'étude de l'itération des transformations continues, Math. Zeit. vol. 48 (1943) pp. 685-711.

31. S. Fomin, Finite invariant measures in the flows, Rec. Math. (Mat. Sbornik) N.S. vol. 12 (1943) pp. 99-108.

32. M. Fréchet, Sur le thêorème ergodique de Birkhoff, C. R. Acad. Sci. Paris vol. 213 (1941) pp. 607-609.

33. - Sur le problème ergodique, La Revue Scientifique (Revue Rose I1lustrée) vol. 81 (1943) pp. 155-157.

34. - - Une application des fonctions asymptotiquement presque-périodiques a l'étude des familles de transformations ponctuelles et au problème ergodique, La Revue Scientifique (Revue Rose Illustrée) vol. 79 (1941) pp. 407-417. 
35. M. Fukamiya, On dominated ergodic theorems in $L_{p}(p \geqq 1)$, Tôhoku Math. J. vol. 46 (1940) pp. 150-153.

36. P. R. Halmos, The decomposition of measures, Duke Math. J. vol. 8 (1941) pp. 386-392.

37. - Square roots of measure preserving transformations, Amer. J. Math. vol. 64 (1942) pp. 153-166.

38. P. R. Halmos and J. von Neumann, Operator methods in classical mechanics, II, Ann. of Math. vol. 43 (1942) pp. 332-350.

39. P. R. Halmos, On automorphisms of compact groups, Bull. Amer. Math. Soc. vol. 49 (1943) pp. 619-624.

40. - Approximation theories for measure preserving transformations, Trans. Amer. Math. Soc. vol. 55 (1944) pp. 1-18.

41. - In general a measure preserving transformation is mixing, Ann. of Math. vol. 45 (1944) pp. 786-792.

42. ——, An ergodic theorem, Proc. Nat. Acad. Sci. U.S.A. vol. 32 (1946) pp. 156-161.

43. - Invariant measures, Ann. of Math. vol. 48 (1947) pp. 735-754.

44. - On a theorem of Dieudonné, Proc. Nat. Acad. Sci. U.S.A. vol. 35 (1949) pp. 38-42.

45. - - A non homogeneous ergodic theorem, Trans. Amer. Math. Soc. vol. 66 (1949) pp. 284-288.

46. —, Measure theory, New York, 1949.

47. P. Hartman and A. Wintner, Asymptotic distributions and the ergodic theorem, Amer. J. Math. vol. 61 (1939) pp. 977-984.

48. - Statistical independence and statistical equilibrium, Amer. J. Math. vol. 62 (1940) pp. 646-654.

49. - Integrability in the large and dynamical stability, Amer. J. Math. vol. 65 (1943) pp. 273-278. 199.

50. P. Hartman, On the ergodic theorems, Amer. J. Math. vol. 69 (1947) pp. 193-

51. G. A. Hedlund, On the metrical transitivity of the geodesics on a surface of constant negative curvature, Proc. Nat. Acad. Sci. U.S.A. vol. 20 (1934) pp. 136-140.

52. - On the metrical transitivity of the geodesics on closed surfaces of constant negative curvature, Ann. of Math. vol. 35 (1934) pp. 787-808.

53. - A metrically transitive group defined by the modular group, Amer. J. Math. vol. 57 (1935) pp. 668-678.

54. - The dynamics of geodesic flows, Bull. Amer. Math. Soc. vol. 45 (1939) pp. $241-260$. 383.

55. —, Fuchsian groups and mixtures, Ann. of Math. vol. 40 (1939) pp. 370-

56. — A new proof for a metrically transitive system, Amer. J. Math. vol. 62 (1940) pp. 233-242.

57. H. Hilmy, Sur le thêorème ergodique, C. R. (Doklady) Acad. Sci. URSS vol. 24 (1939) pp. 213-216.

58. — - Sur le récurrence ergodique dans les systèmes dynamiques, Rec. Math. (Mat. Sbornik) N.S. vol. 7 (1940) pp. 101-109.

59. E. Hopf, Zwei Sätze über den wahrscheinlichen Verlauf der Bewegungen dynamischer Systeme, Math. Ann. vol. 103 (1930) pp. 710-719.

60. - On the time average theorem in dynamics, Proc. Nat. Acad. Sci. U.S.A. vol. 18 (1932) pp. 93-100.

61. - U Über lineare Gruppen unitärer Operatoren im Zusammenhange mit den 
Bewegungen dynamischer Systeme, Sitzungsberichte der Preussischen Akademie der Wissenschaften vol. 14 (1932) pp. 182-190.

62. - Complete transitivity and the ergodic principle, Proc. Nat. Acad. Sci. U.S.A. vol. 18 (1932) pp. 204-209.

63. - Proof of Gibbs' hypothesis on the tendency toward statistical equilibrium, Proc. Nat. Acad. Sci. U.S.A. vol. 18 (1932) pp. 333-340.

64. - Theory of measure and invariant integrals, Trans. Amer. Math. Soc. vol. 34 (1932) pp. 373-393.

65. - On causality, statistics, and probability, Journal of Mathematics and Physics vol. 13 (1934) pp. 51-102.

66. - Fuchsian groups and ergodic theory, Trans. Amer. Math. Soc. vol. 39 (1936) pp. 299-314.

67. —, Ergodentheorie, Berlin, 1937.

68. - Statistische Probleme und Ergebnisse in der klassischen Mechanik, Actualités Scientifiques et Industrielles, no. 737, 1938, pp. 5-16.

69. - Beweis des Mischungscharakters der geodätischen Strömung auf Flächen der Krïmmung minus Eins und endlicher Oberfläche, Sitzungsberichte der Preussischen Akademie der Wissenschaften (1938) pp. 333-344.

70. - - Statistik der geodätischen Linien in Mannigfaltigkeiten negativer Kriummung, Berichte über die Verhandlungen der Sächsischen Akademie der Wissenschaften zu Leipzig vol. 91 (1939) pp. 261-304.

71. - Statistik der Lösungen geodätischen Probleme vom unstabilen Typus, II, Math. Ann. vol. 117 (1940) pp. 590-608.

72. — Über eine Ungleichung der Ergodentheorie, Sitzungsberichte der Mathematisch-Naturwissenschaftlichen Klasse der Bayerischen Akademie der Wissenschaften zu München (1944) pp. 171-176.

73. W. Hurewicz, Ergodic theorem without invariant measure, Ann. of Math. vol. 45 (1944) pp. 192-206.

74. K. Ito, On the ergodicity of a certain stationary process, Proc. Imp. Acad. Tokyo vol. 20 (1944) pp. 54-55.

75. S. Izumi, A non-homogeneous ergodic theorem, Proc. Imp. Acad. Tokyo vol. 15 (1939) pp. 189-192.

76. - A remark on ergodic theorems, Proc. Imp. Acad. Tokyo vol. 19 (1943) pp. $102-104$.

77. B. Jessen, Abstract theory of measure and integration. IX, Matematisk Tidsskrift. B (1947) pp. 21-26.

78. - Abstrakt Maal-og Integralteori, Copenhagen, 1947.

79. M. Kac, On the notion of recurrence in discrete stochastic processes, Bull. Amer. Math. Soc. vol. 53 (1947) pp. 1002-1010.

80. S. Kakutani and K. Yosida, Birkhoff's ergodic theorem and the maximal ergodic theorem, Proc. Imp. Acad. Tokyo vol. 15 (1939) pp. 165-168.

81. S. Kakutani, Representation of measurable flows in Euclidean 3-space, Proc. Nat. Acad. Sci. U.S.A. vol. 28 (1942) pp. 16-21.

82. - Induced measure preserving transformations, Proc. Imp. Acad. Tokyo vol. 19 (1943) pp. 635-641.

83. Y. Kawada, Über die masstreuen Abbildungen vom Mischungstypus im weiteren Sinne, Proc. Imp. Acad. Tokyo vol. 19 (1943) pp. 520-524.

84. - Über die masstreuen Abbildungen in Produkträumen, Proc. Imp. Acad. Tokyo vol. 19 (1943) pp. 525-527.

85. —- Über die Existenz der invarianten Integrale, Jap. J. Math. vol. 19 (1944) pp. $81-95$. 
86. A. Khintchine, Zur mathematischen Begrilndung der statistischen Mechanik, Zeitschrift für Angewandte Mathematik und Mechanik, vol. 13 (1933) pp. 101-103.

87. - The method of spectral reduction in classical dynamics, Proc. Nat. Acad. Sci. U.S.A. vol. 19 (1933) pp. 567-573.

88. - Zu Birkhoffs Lösung des Ergodenproblems, Math. Ann. vol. 107 (1933) pp. $485-488$.

89. - Fourierkoeffizienten längs einer Bahn im Phasenraum, Rec. Math. (Mat. Sbornik) vol. 41 (1934) pp. 14-15.

90. - Eine Verschärfung des Poincaréschen "Wiederkehrsatzes," Compositio Math. vol. 1 (1934) pp. 177-179.

91. K Korrelationstheorie der stationären stochastischen Prozesse, Math. Ann. vol. 109 (1934) pp. 604-615.

92. A. Kolmogoroff, Ein vereinfachter Beweis des Birkhoff-Khintchineschen Ergodensatzes, Rec. Math. (Mat. Sbornik) N.S. vol. 2 (1937) pp. 366-368.

93. B. O. Koopman, Hamiltonian systems and transformations in Hilbert space, Proc. Nat. Acad. Sci. U.S.A. vol. 17 (1931) pp. 315-318.

94. B. O. Koopman and J. von Neumann, Dynamical systems of continuous spectra, Proc. Nat. Acad. Sci. U.S.A. vol. 18 (1932) pp. 255-263.

95. T. Levi-Civita, $A$ general survey of the theory of adiabatic invariants, Journal of Mathematics and Physics vol. 13 (1934) pp. 18-40.

96. F. Maeda, Application of the theory of set functions to the mixing of fluids, Journal of Science of the Hirosima University. Ser. A vol. 5 (1935) pp. 1-6.

97. - Transitivities of conservative mechanism, Journal of Science of the Hirosima University. Ser. A vol. 6 (1936) pp. 1-18.

98. D. Maharam, On homogeneous measure algebras, Proc. Nat. Acad. Sci. U.S.A. vol. 28 (1942) pp. 108-111.

99. P. T. Maker, The ergodic theorem for a sequence of functions, Duke Math. J. vol. 6 (1940) pp. 27-30.

100. M. H. Martin, Metrically transitive point transformations, Bull. Amer. Math. Soc. vol. 40 (1934) pp. 606-612.

101. J. von Neumann, Proof of the quasi-ergodic hypothesis, Proc. Nat. Acad. Sci. U.S.A. vol. 18 (1932) pp. 70-82.

102. - Einige Sätze ilber messbare Abbildungen, Ann. of Math. vol. 33 (1932) pp. 574-586.

103. - Zur Operatorenmethode in der klassischen Mechanik, Ann. of Math. vol. 33 (1932) pp. 587-642.

104. - Zusätze zur Arbeit "Zur Operatorenmethode....," Ann. of Math. vol. 33 (1932) pp. 789-791.

105. J. C. Oxtoby, Note on transitive transformations, Proc. Nat. Acad. Sci. U.S.A. vol. 23 (1937) pp. 443-446.

106. J. C. Oxtoby and S. M. Ulam, On the existence of a measure invariant under a transformation, Ann. of Math. vol. 40 (1939) pp. 560-566.

107. - Measure-preserving homeomorphisms and metrical transitivity, Ann. of Math. vol. 42 (1941) pp. 874-920.

108. J. C. Oxtoby, On the ergodic theorem of Hurewicz, Ann. of Math. vol. 49 (1948) pp. 872-884.

109. H. R. Pitt, Some generalizations of the ergodic theorem, Proc. Cambridge Philos. Soc. vol. 38 (1942) pp. 325-343.

110. H. Rademacher, Eineindeutige Abbildungen und Messbarkeit, Monatshefte für Mathematik und Physik vol. 27 (1916) pp. 183-290. 
111. F. Riesz, Sur quelques problèmes de la thêorie ergodique, Matematikai és Fizikai Lapok vol. 49 (1942) pp. 34-62.

112. — - Sur la theorie ergodique, Comment. Math. Helv. vol. 17 (1945) pp. 221-239.

113. V. Rokhlin, On the classification of measurable decompositions, Doklady Akademii Nauk SSSR vol. 58 (1947) pp. 29-32.

114. - On the problem of the classification of automorphisms of Lebesgue spaces, Doklady Akademii Nauk SSSR vol. 48 (1947) pp. 189-191.

115. — - A "general" measure preserving transformation is not mixing, Doklady Akademii Nauk SSSR vol. 60 (1948) pp. 349-351.

116. - - Unitary rings, Doklady Akademii Nauk SSSR vol. 59 (1948) pp. 643-646.

117. G. Scorza Dragoni, Sul teorema ergodico, Rend. Circ. Mat. Palermo vol. 58 (1934) pp. 311-325.

118. —- Transitivita metrica e teoremi di media, Rend. Circ. Mat. Palermo vol. 59 (1935) pp. 235-255.

119. - Sul fondamento matematico della teoria degli invarianti adiabatici, Annali di Mathematica Pura ed Applicata vol. 13 (1935) pp. 335-362.

120. W. Seidel, Note on a metrically transitive system, Proc. Nat. Acad. Sci. U.S.A. vol. 19 (1933) pp. 453-456.

121. - On a metric property of Fuchsian groups, Proc. Nat. Acad. Sci. U.S.A. vol. 21 (1935) pp. 475-478.

122. W. Stepanoff, Sur une extension du thêorème ergodique, Compositio Math. vol. 3 (1936) pp. 239-253.

123. M. Tsuji, On Hopf's ergodic theorem, Proc. Imp. Acad. Tokyo vol. 20 (1944) pp. $640-647$.

124. - Some metrical theorems on Fuchsian groups, Proc. Imp. Acad. Tokyo vol. 21 (1945) pp. 104-109.

125. - On Hopf's ergodic theorem, Jap. J. Math. vol. 19 (1945) pp. 259-284.

126. C. Visser, On Poincarê's recurrence theorem, Bull. Amer. Math. Soc. vol. 42 (1936) pp. 397-400.

127. N. Wiener, The homogeneous chaos, Amer. J. Math. vol. 60 (1938) pp. 897936.

128. - - The ergodic theorem, Duke Math. J. vol. 5 (1939) pp. 1-18.

129. N. Wiener and A. Wintner, Harmonic analysis and ergodic theory, Amer. J. Math. vol. 63 (1941) pp. 415-426.

130. - On the ergodic dynamics of almost periodic systems, Amer. J. Math. vol. 63 (1941) pp. 794-824.

131. - The discrete chaos, Amer. J. Math. vol. 65 (1943) pp. 279-298.

132. A. Wintner, Remarks on the ergodic theorem of Birkhoff, Proc. Nat. Acad. Sci. U.S.A. vol. 18 (1932) pp. 248-251.

133. —, Dynamische Systeme und unitäre Matrizen, Math. Zeit. vol. 36 (1933) pp. $630-637$.

134. - On the ergodic analysis of the remainder term of mean motions, Proc. Nat. Acad. Sci. U.S.A. vol. 26 (1940) pp. 126-129.

135. K. Yosida, Ergodic theorems of Birkhoff-Khintchine's type, Jap. J. Math. vol. 17 (1940) pp. 31-36.

136. - , An abstract treatment of the individual ergodic theorem, Proc. Imp. Acad. Tokyo vol. 16 (1940) pp. 280-284.

University of Chicago 